\title{
Monitoramento de mosca-minadora Liriomyza sp. (Diptera:Agromyzidae) em meloeiro e levantamento de seus inimigos naturais no município de Floresta, Pernambuco Monitoring of leafminers Liriomyza sp. (Diptera: Agromyzidae) in melon and verification of their natural enemies in the municipality of Floresta, Pernambuco
}

\author{
FERRAZ, Natália de Menezes Gomes. Técnica em Agropecuária \\ IF Sertão-PE - Floresta - PE - Brasil. / E-mail: e-mail_do_autor@ifsertao-pe.edu.br
}

\section{RESUMO}

Dentre os insetos-pragas que limitam a produtividade do meloeiro estão as moscas-minadoras do gênero Liriomyza. A maioria dos produtores adotam o controle químico para reduzir a incidência dessa praga nos plantios, porém o uso indiscriminado desse método pode causar vários problemas de ordem ecológica, econômica e social. Uma alternativa para o manejo desta praga é o controle biológico com o uso de parasitoides. Portanto, este trabalho teve como objetivos: identificar a espécie mais importante de mosca-minadora para o município de Floresta-PE; monitorar a sua densidade populacional durante todo o ciclo da cultura; verificar a ocorrência de inimigos naturais (parasitoides), bem como sua taxa de parasitismo para seleção dos mais promissores e verificar a melhor época de liberação conforme a fenologia da cultura. Os experimentos foram conduzidos em duas áreas comerciais de meloeiro localizadas nos arredores de Floresta-PE, onde selecionou-se, de forma randomizada, 30 plantas para amostragem de larvas presentes nas folhas. Foram analisadas 30 folhas para verificação da emergência, tanto de Liriomyza quanto de parasitoides, para posterior identificação e contabilização. Os levantamentos ocorreram semanalmente até a colheita e totalizaram sete por área. Os dados das amostragens de minadora foram submetidos à análise de variância e ao teste de Tukey (5\%). Os dados da taxa de parasitismo foram sujeitos a uma fórmula específica. De acordo com os resultados obtidos, a espécie de mosca-minadora que predomina na localidade em questão é Liriomyza sativae, sendo a sua maior ocorrência nos estágios iniciais da cultura do meloeiro. Dentre os inimigos naturais, os mais abundantes e com maior taxa de parasitismo foram os da família Eulophidae e a melhor época de liberação dos mesmos é entre a segunda e a terceira semana após o plantio.

Palavras-chave: Amostragem, Controle biológico, Melão.

\section{ABSTRACT}

Among the insect that limit the productivity of the melon are the leafminers of the genus Liriomyza. Most producers adopt chemical control to reduce the incidence of this pest in crops, however the indiscriminate use of this method can cause several ecological, economic and social problems. An alternative to the management of this pest is the biological control with the use of parasitoids. Therefore, this work had as objectives: to identify the most important species of leafminer for the municipality of Floresta-PE; monitor their population density throughout the crop cycle; to verify the occurrence of natural enemies (parasitoids), as well as their parasitism rate to select the most promising ones and to check the best liberation time according to the culture phenology. The experiments were conducted in two commercial melon areas located in the proximity of Floresta-PE, where 30 plants were randomly selected for sampling of larvae present on the leaves. Thirty leaves were analyzed to verify the emergence of Liriomyza and parasitoids for later to realize the identification and the counting. The surveys occurred weekly until harvest and totaled seven per area. The data from the samples were submitted to analysis of variance and to the Tukey's test (5\%). The parasitism rate data were subjected to a specific formula. According to the results obtained, the species of leafminer that predominates in the locality is Liriomyza sativae, being its greater occurrence in the initial phases of the melon culture. Among the natural enemies, the most abundant and with the highest rate of parasitism were those of the Eulophidae family and the best time for their release is between the second and third week after planting.

Keywords: Sampling, Biological control, Melon. 
FERRAZ, N. M. G.

Monitoramento de mosca-minadora Liriomyza sp. (Diptera: Agromyzidae) em meloeiro e levantamento de seus inimigos naturais no município de Floresta, Pernambuco

\section{Introdução}

O melão (Cucumis melo L.) é um fruto muito apreciado e de grande popularidade no mundo, sendo, atualmente, uma das frutas frescas mais exportadas pelo Brasil (NASCIMENTO NETO, 2011). Somente no ano de 2007, o valor exportado ficou em torno de US\$ 128 milhões, e em 2008 elevou-se para US\$ 152 milhões, garantindo à cultura do meloeiro o primeiro lugar no ranking de exportações no ano de 2009 (LIMA, 2012).

A maior parte da produção nordestina de melão é exportada para a União Européia e para o Reino Unido (SALES JÚNIOR et al., 2006). Apesar do cenário favorável ao cultivo do meloeiro, os produtores do semiárido, nos últimos anos, têm enfrentado sérios problemas com o ataque da moscaminadora Liriomyza spp. que tem limitado a produtividade da cultura (ARAUJO et al., 2007).

De acordo com Murphy \& Lasalle (1999), existem três espécies de mosca-minadora pertencentes ao gênero Liriomyza que são de grande importância econômica, pois são cosmopolitas (ampla distribuição mundial) e polífagas (suas larvas se alimentam de várias espécies vegetais, portanto, têm importância agrícola), são elas: L. trifolii (Burgess, 1880), L. sativae (BLANCHARD, 1938 e L. huidobrensis (BLANCHARD, 1926). Os danos ocasionados por estes insetos podem ser classificados em diretos, quando há a perfuração da folha para deposição dos ovos e através da mina causada pela larva durante o processo de desenvolvimento e alimentação do mesófilo foliar, e indiretos, causando diminuição da área foliar (reduzindo a atividade fotossintética), depreciação do valor comercial do produto, pontos de entrada para patógenos, "stress" hídrico, atrofiamento e até morte das plantas (MUSGRAVE et al.,1975). Os prejuízos em decorrência do ataque da praga são enormes, o que provoca o aumento do custo de produção com a utilização de inseticidas (ARAÚJO et al., 2007).

Atualmente, o método de controle mais utilizado para mosca-minadora em meloeiro é o químico através do uso de inseticidas, porém há poucos ingredientes ativos registrados no Ministério da Agricultura, Pecuária e Abastecimento (MAPA) para controle da referida praga em meloeiro (AGROFIT, 2020). Além de dificultar o seu controle, pois o número limitado de produtos registrados dificulta a rotação de princípios ativos, isso acaba criando um cenário propício ao desenvolvimento de resistência desta praga a inseticidas (GUIMARÃES et al., 2009). Portanto, faz-se necessária a busca por práticas de controle para a mosca-minadora, adequando-as à diversidade de ambientes, ao sistema de cultivo e, principalmente, ao nível tecnológico dos produtores locais. Nesse contexto, o controle biológico com o uso de inimigos naturais (predadores e parasitoides) é uma alternativa bastante viável, uma vez que atua na regulação populacional da praga sem causar nenhum risco ao ambiente (PARRA et al. 2002). Segundo estes mesmos autores, a vantagem do uso de agentes de controle biológico, em detrimento ao controle químico, está no fato de os inimigos naturais não fazerem distinção entre indivíduos resistentes e susceptíveis a defensivos químicos, regulando suas populações a níveis toleráveis.

Apesar da grande importância econômica da mosca-minadora para várias culturas no Brasil e do potencial de ação de seus inimigos naturais, ainda são escassos os relatos sobre ambos no país (COSTA-LIMA et al., 2015), principalmente na região Nordeste (ARAUJO et al., 2007), e o cenário fica 
FERRAZ, N. M. G.

Monitoramento de mosca-minadora Liriomyza sp. (Diptera: Agromyzidae) em meloeiro e levantamento de seus inimigos naturais no município de Floresta, Pernambuco

mais crítico quando se trata do município de Floresta-PE. Diante disso, este trabalho teve como objetivos: (I) identificar a espécie mais importante de mosca-minadora para o município de FlorestaPE; (II) monitorar a sua densidade populacional durante todo o ciclo da cultura; (III) verificar a ocorrência de inimigos naturais (parasitoides), bem como sua taxa de parasitismo para seleção dos mais promissores e (IV) verificar a melhor época de liberação dos parasitoides de acordo com a fenologia da cultura.

\section{Material e métodos}

O trabalho foi conduzido em duas áreas de plantios comerciais de meloeiro situadas aos arredores do município de Floresta-PE. Os levantamentos dos insetos foram realizados semanalmente, durante todo o ciclo da cultura, e consistiu na coleta de, no mínimo, 30 folhas apresentando minas ocasionadas por mosca-minadora. As folhas coletadas foram acondicionadas em bandejas plásticas, cobertas com tecido organza e mantidas sob temperatura ambiente $\left(25^{\circ} \pm 2^{\circ} \mathrm{C}\right)$ até a emergência das moscas-minadoras adultas e/ou dos seus parasitoides. Após isso, ambos foram contabilizados e identificados.

As espécies de moscas-minadoras foram reconhecidas com base na chave de identificação de Spencer \& Steyskal (1986) e pelas características das injúrias deixadas nas folhas (Central Science Laboratory, UK, 2004). Já os seus parasitoides foram identificados a partir dos caracteres morfológicos externos apresentados em La Salle \& Parrela (1991) e Wharton et al. (1997).

Para a amostragem de mosca-minadora, selecionou-se trinta plantas ao acaso em campo e bem distribuídas para uma melhor casualização, onde foi contado o número de minas ativas (com larvas vivas em seu interior) nas folhas. Esses dados foram analisados da seguinte forma: o número de larvas/minas por folha foi submetido à análise de variância e as médias comparadas pelo teste de Tukey. Isso foi efetuado com o intuito de verificar a diferença de infestação de mosca-minadora no decorrer do ciclo da cultura, bem como ver em qual estágio fenológico ocorre o maior ataque da referida praga. Para a análise, foi utilizado o software SAS.

Já o percentual de parasitismo foi calculado com base na fórmula: $\left(\mathrm{n}^{\circ}\right.$ de parasitóides obtidos / $\mathrm{n}^{\circ}$ de pupários da mosca-minadora) x 100, como indicado por Guimarães et al. (2010), sendo que a espécie de parasitoide que apresentou a maior taxa de parasitismo foi considerada como a ideal para ser usada em programas de controle biológico de mosca-minadora no município de Floresta-PE.

\section{Resultados e discussão}

\section{Identificação da mosca-minadora de maior importância para Floresta-PE}

Foram coletados 812 espécimes de mosca-minadora que emergiram dos pupários obtidos das folhas de meloeiro coletadas no campo nas duas áreas de levantamento (Tabelas 1 e 2). Todos pertenciam a espécie Liriomyza sativae, o que já era esperado, uma vez que esta espécie é a de maior ocorrência em regiões com temperatura elevada (GUIMARÃES et al., 2009). Levantamentos de 
FERRAZ, N. M. G.

Monitoramento de mosca-minadora Liriomyza sp. (Diptera: Agromyzidae) em meloeiro e levantamento de seus inimigos naturais no município de Floresta, Pernambuco

moscas-minadoras foram realizados em várias localidades do Nordeste brasileiro por Ramalho e Moreira (1979), podendo ser observada a presença de L. sativae nas culturas do meloeiro, melancieira, tomateiro e cravo-de-defunto (Tagetes patula L.).

Ferreira (2014), mediante caracterização molecular, identificou também L. sativae em populações coletadas em meloeiro no Rio Grande do Norte, Ceará e Bahia. 0 mesmo autor também verificou $L$. sativae associado a tomateiro, em Pernambuco e Espírito Santo, e feijoeiro, também em Pernambuco. De acordo com Fernandes (2004) e Guimarães et al. (2008), nos polos produtores de melão localizados no Nordeste brasileiro, principalmente em Mossoró e Assu, RN, as espécies de moscas-minadoras predominantes são $L$. sativae e $L$. trifolii. Embora nas bordaduras das áreas estudadas em Floresta-PE houvesse plantas de feijoeiro, não foi detectada a presença de L. trifolii, a qual também tem maior ocorrência em regiões de temperaturas elevadas. Portanto, de acordo com as amostragens realizadas, pode-se inferir que $L$. sativae é a espécie de mosca-minadora de maior importância para os cultivos de meloeiro do município.

Tabela 1. Número de espécimes de L. sativae, Opius sp. (Hymenoptera: Braconidae) e parasitoides da família Eulophidae obtidos de folhas de meloeiro coletadas em plantio comercial no munícipio de Floresta-PE no período de 21/02 a 06/04/2017 (área 1).

\begin{tabular}{cccc}
\hline Data da coleta & L. sativae & Eulophidae & Opius sp. \\
\hline $21 / 02 / 2017$ & 153 & 108 & 0 \\
$02 / 03 / 2017$ & 152 & 128 & 4 \\
$07 / 03 / 2017$ & 162 & 89 & 5 \\
$14 / 03 / 2017$ & 29 & 37 & 8 \\
$22 / 03 / 2017$ & 6 & 9 & 5 \\
$28 / 03 / 2017$ & 1 & 0 & 0 \\
$06 / 04 / 2017$ & 0 & 0 & 0 \\
\hline Total & 503 & 371 & 22 \\
\hline
\end{tabular}

Tabela 2. Número de espécimes de L. sativae, Opius sp. (Hymenoptera: Braconidae) e parasitoides da família Eulophidae obtidos de folhas de meloeiro coletadas em plantio comercial no munícipio de Floresta-PE no período de 11/04 a 23/05/2017 (área 2).

\begin{tabular}{cccc}
\hline Data da coleta & L. sativae & Eulophidae & Opius sp. \\
\hline $11 / 04 / 2017$ & 97 & 19 & 9 \\
$18 / 04 / 2017$ & 39 & 17 & 0 \\
$25 / 04 / 2017$ & 47 & 21 & 9 \\
$03 / 05 / 2017$ & 64 & 6 & 6 \\
$09 / 05 / 2017$ & 21 & 6 & 4 \\
$16 / 05 / 2017$ & 39 & 11 & 4 \\
$23 / 05 / 2017$ & 2 & 3 & 2 \\
\hline Total & 309 & 83 & 34 \\
\hline
\end{tabular}

Monitoramento de $L$. sativae durante todo o ciclo da cultura

Os picos de ocorrência de $L$. sativae nos plantios não variaram muito entre as áreas. $\mathrm{Na}$ área 
FERRAZ, N. M. G.

Monitoramento de mosca-minadora Liriomyza sp. (Diptera: Agromyzidae) em meloeiro e levantamento de seus inimigos naturais no município de Floresta, Pernambuco

1, a maior infestação desta praga ocorreu entre a segunda e terceira amostragem, entre 23 a 38 dias após o plantio (Figura 1; Tabela 3). Enquanto que, na área 2, o maior pico de ocorrência da praga foi na primeira amostragem, 14 D.A.P. (Figura 2; Tabela 4), portanto, ambas ocorreram na fase vegetativa da cultura, antes do florescimento. Entretanto, alguns estudos com esta espécie de mosca-minadora não encontraram influência da fenologia da planta em sua flutuação populacional. Por exemplo, Alencar et al. (2002) ao estudarem a dinâmica populacional de L. sativae na cultura do melão no semiárido nordestino, verificaram a influência da temperatura, mas não detectaram a influência da fenologia da planta. O mesmo ocorre em outros trabalhos (PALUMBO et al., 1994; ZHANG et al., 2000; COSTA LIMA et al., 2009).

Leite et al. (2004) e Azevedo et al. (2005) verificaram que a maior ocorrência de moscasminadoras acontece no final de ciclo (fase reprodutiva), entretanto, não avaliaram a influência de nenhum fator biótico ou abiótico sobre a flutuação populacional desses insetos, podendo-se inferir que os fatores climáticos são o que realmente regulam a dinâmica populacional. Além disso, o fator Fenologia teria mais importância se os insetos estudados atacassem estruturas reprodutivas de plantas, o que não é o caso de L. sativae, uma vez que se trata de uma praga foliar a qual conta com disponibilidade de folhas durante todo o ciclo do meloeiro.

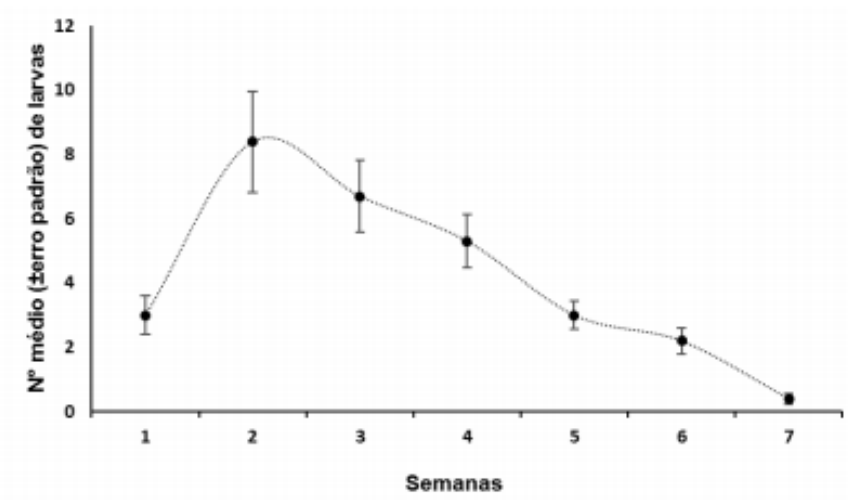

Figura 1. Flutuação populacional (número médio de larvas/minas de Liriomyza sativae presentes na $12^{\circ}$ folha) em meloeiro no período de $21 / 02$ a 06/04/2017 no município de Floresta -PE (área 1).

Tabela 3. Número médio de larvas/minas de L. sativae em 30 plantas de meloeiro no decorrer do ciclo da cultura (área 1).

\begin{tabular}{ccccc}
\hline Data & $\begin{array}{c}\text { DAP (dias } \\
\text { após o } \\
\text { plantio) }\end{array}$ & Fenologia da planta & $\begin{array}{c}\mathbf{N}^{\mathbf{0}} \text { da } \\
\text { Amostragem }\end{array}$ & $\begin{array}{c}\mathbf{N}^{\mathbf{0}} \text { médio de } \\
\text { larvas/minas de } \boldsymbol{L} . \\
\text { sativae }\end{array}$ \\
\hline $07 / 02 / 2017$ & 0 & - & Plantio & - \\
$14 / 02 / 2017$ & 7 & Fase vegetativa & - & - \\
$21 / 02 / 2017$ & 14 & Fase vegetativa & $1^{\text {o }}$ amostragem & $3,0 \pm 0,61 \mathrm{bc}$ \\
$02 / 03 / 2017$ & 23 & Fase vegetativa & $2^{\circ}$ amostragem & $8,4 \pm 1,57^{\mathrm{a}}$ \\
$07 / 03 / 2017$ & 28 & Florescimento & $3^{\circ}$ amostragem & $6,7 \pm 1,11^{\mathrm{a}}$ \\
$14 / 03 / 2017$ & 35 & Florescimento/frutificação & $4^{\circ}$ amostragem & $5,3 \pm 0,83 \mathrm{ab}$ \\
$22 / 03 / 2017$ & 43 & Frutificação & $5^{\circ}$ amostragem & $3,0 \pm 0,45 \mathrm{bc}$ \\
$28 / 03 / 2017$ & 49 & Maturação & $6^{\circ}$ amostragem & $2,2 \pm 0,40 \mathrm{bc}$ \\
$06 / 04 / 2017$ & 58 & Senescência & $7^{\circ}$ amostragem & $0,4 \pm 0,16 \mathrm{c}$ \\
$11 / 04 / 2017$ & 63 & Colheita & - & - \\
\hline
\end{tabular}

Médias seguidas de mesma letra na mesma coluna não diferem significativamente entre si, pelo teste de Tukey $(P \geq 0,05)$. 


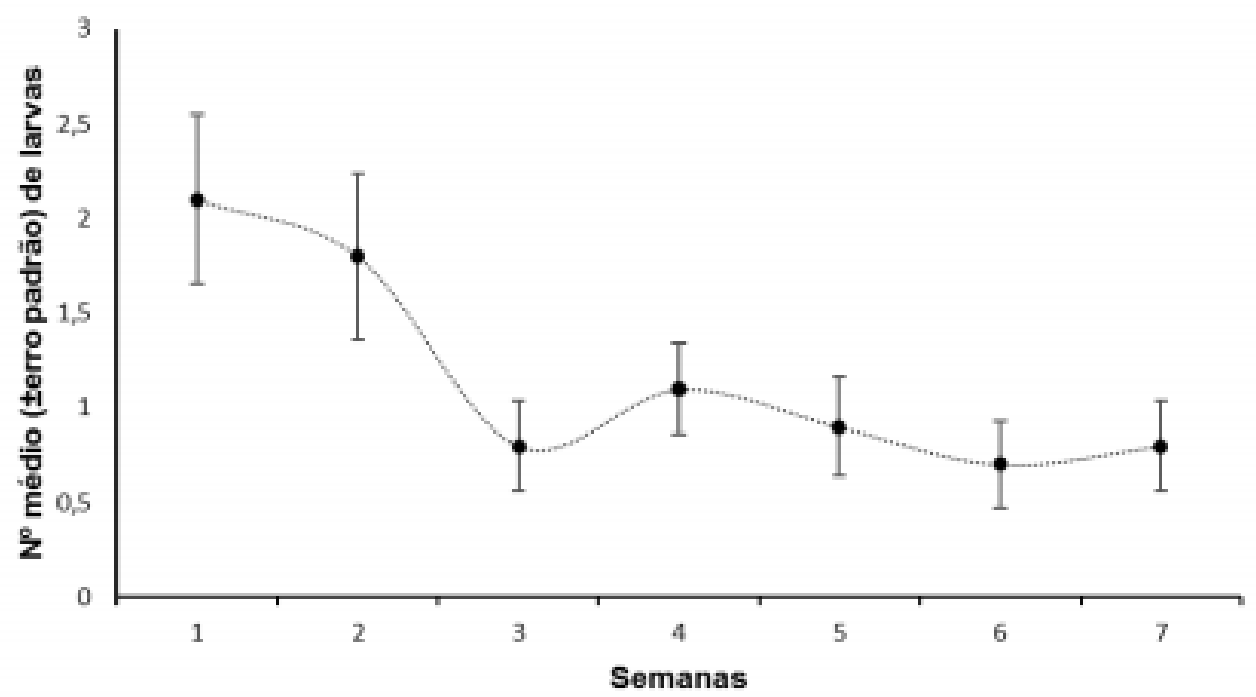

Figura 2. Flutuação populacional (número médio de larvas/minas de Liriomyza sativae presentes na $12^{\circ}$ folha) em meloeiro no período de 11/04 a 23/05/2017 no município de Floresta -PE (área 2).

Tabela 4. Número médio de larvas/minas de L. sativae em 30 plantas de meloeiro no decorrer do ciclo da cultura (área 2).

\begin{tabular}{ccccc}
\hline Data & $\begin{array}{c}\text { DAP (dias } \\
\text { após o } \\
\text { plantio) }\end{array}$ & Fenologia da planta & $\begin{array}{c}\mathbf{N}^{\mathbf{0}} \text { da } \\
\text { Amostragem }\end{array}$ & $\begin{array}{c}\mathbf{N}^{\mathbf{0}} \text { médio de } \\
\text { larvas/minas de } \boldsymbol{L} . \\
\text { sativae }\end{array}$ \\
\hline $28 / 03 / 2017$ & 0 & - & Plantio & - \\
$04 / 04 / 2017$ & 7 & Fase vegetativa & - & - \\
$11 / 04 / 2017$ & 14 & Fase vegetativa & $1^{\text {o }}$ amostragem & $2,1 \pm 0,45 \mathrm{a}$ \\
$18 / 04 / 2017$ & 21 & Fase vegetativa & $2^{\mathbf{o}}$ amostragem & $1,8 \pm 0,44 \mathrm{ab}$ \\
$25 / 04 / 2017$ & 28 & Florescimento & $3^{\circ}$ amostragem & $0,8 \pm 0,24 \mathrm{ab}$ \\
$03 / 05 / 2017$ & 36 & Florescimento/frutificação & $4^{\circ}$ amostragem & $1,1 \pm 0,24 \mathrm{ab}$ \\
$09 / 05 / 2017$ & 42 & Frutificação & $5^{\circ}$ amostragem & $0,9 \pm 0,26 \mathrm{ab}$ \\
$16 / 05 / 2017$ & 49 & Maturação & $6^{\circ}$ amostragem & $0,7 \pm 0,23 \mathrm{~b}$ \\
$23 / 05 / 2017$ & 56 & Senescência & $7^{\circ}$ amostragem & $0,8 \pm 0,24 \mathrm{~b}$ \\
$29 / 05 / 2017$ & 62 & Colheita & - & - \\
\hline
\end{tabular}

Médias seguidas de mesma letra na mesma coluna não diferem significativamente entre si, pelo teste de Tukey $(P \geq 0,05)$.

\section{Ocorrência de parasitoides e taxa de parasitismo dos mesmos sobre L. sativae}

Em relação a emergência de parasitoides dos pupários de $L$. sativae obtidos, emergiram parasitoides das famílias Braconidae e Eulophidae de Hymenoptera nas duas áreas, o que está de acordo com as revisões de Murphy \& LaSalle (1999), nas quais os autores indicam essas famílias como as principais representantes de parasitoides de moscas-minadoras do gênero Liriomyza.

$\mathrm{Na}$ área 1, foram identificados 22 espécimes de parasitoides da família Braconidae, sendo todos do gênero Opius e 371 da família Eulophidae, cuja identificação a nível taxonômico mais específico não foi possível, porém acredita-se que pertença ao gênero Neochrysocharis. Ainda assim, é preciso que os mesmos sejam enviados para um especialista no grupo para uma identificação mais precisa. 
FERRAZ, N. M. G

Monitoramento de mosca-minadora Liriomyza sp. (Diptera: Agromyzidae) em meloeiro e levantamento de seus inimigos naturais no município de Floresta, Pernambuco

O gênero Opius já foi registrado em todas as regiões do Brasil em que houveram levantamentos, ou seja, nordeste (ARAÚJO et al., 2007), centro-oeste (GUIMARÃES et al., 2010), sudeste (CRUZ et al., 1988) e sul (RIBEIRO et al., 2007). Já Neochrysocharis sp. são apontados em diversos estudos como alguns dos principais inimigos naturais de mosca-minadora, principalmente devido ao alto índice de parasitismo e à alta frequência desses parasitoides nos levantamentos populacionais realizados em muitos países (HERNÁNDEZ et al., 2011; STRAKHOVA, 2013; ALBA, 2014), inclusive no Brasil (Costa-Lima et al., 2016).

A maior taxa de parasitismo total (ou seja, durante todo o ciclo) foi obtida pelos parasitoides da família Eulophidae (36,76\%), sendo que, quando se analisou o parasitismo semanal, os mesmos atingiram índices de parasitismos de $100 \%$ na quarta coleta. Em contrapartida, os parasitoides do gênero Opius apresentaram baixa taxa de parasitismo total $(2,18 \%)$, o que indica baixa eficiência desse parasitoide nas condições de Floresta-PE (Tabela 5).

Tabela 5. Porcentagem de parasitismo de dois parasitoides obtidos de pupários de $L$. sativae em folhas de meloeiro coletadas em plantio comercial no munícipio de Floresta-PE no período de 21/02 a 06/04/2017 (área 1).

\begin{tabular}{ccc}
\hline Data da coleta & Eulophidae (\%) & Opius sp. (\%) \\
\hline $21 / 02 / 2017$ & 28,13 & 0 \\
$02 / 03 / 2017$ & 44,60 & 1,39 \\
$07 / 03 / 2017$ & 31,34 & 1,76 \\
$14 / 03 / 2017$ & 100 & 21,62 \\
$22 / 03 / 2017$ & 90 & 50 \\
$28 / 03 / 2017$ & 0 & 0 \\
$06 / 04 / 2017$ & 0 & 0 \\
\hline Total & 36,76 & 2,18
\end{tabular}

Na área 2 (Tabela 6), o cenário repetiu-se, com maior taxa de parasitismo dos parasitoides da família Eulophidae (19,95\%) em comparação a Opius sp. $(8,17 \%)$, o que evidencia a maior eficiência de eulofídeos em relação aos da família Braconidae. Costa (2016) também constatou que as médias de parasitismo do Neochrysocharis sp. foram superiores às de Opius sp. nas safras de meloeiro em Mossoró-RN. No entanto, estes resultados divergem do que se verifica em outros trabalhos, nos quais Opius é mais eficiente do que os parasitoides da família Eulophidae (SILVA, 2008; GUIMARÃES et al. 2010).

Uma das possíveis explicações para isso, deve-se ao fato de que, os métodos de amostragem para parasitoides larva-pupa são difíceis de serem realizados, devido a características intrínsecas deste tipo de parasita (BJORKSTEN et al. 2005). Estes mesmos autores também detectaram uma maior ocorrência de Eulofídeos em comparação a Opius, mesmo havendo uma predominância de Opius sp. no local onde foi realizado o trabalho (Victória, Austrália). É provável que mais estudos sejam conduzidos no município de Floresta-PE para verificar se este padrão se repete em outros anos agrícolas. 
FERRAZ, N. M. G.

Monitoramento de mosca-minadora Liriomyza sp. (Diptera: Agromyzidae) em meloeiro e levantamento de seus inimigos naturais no município de Floresta, Pernambuco

Guimarães et al. (2009) salientaram a falta de informações sobre o parasitismo natural da mosca-minadora em meloeiro e destacaram a importância de mais pesquisas serem realizadas sobre o tema, uma vez que esse conhecimento possibilita o desenvolvimento de programas de controle biológico de pragas em áreas agrícolas, contribuindo para a sustentabilidade do cultivo. No Brasil, ainda não há empresas de controle biológico que comercializem parasitoides de moscas-minadoras, embora haja um crescimento do interesse por esses agentes, principalmente em culturas de alto valor comercial, como melão no nordeste do país (COSTA-LIMA, 2011).

Tabela 6. Porcentagem de parasitismo de dois parasitoides obtidos de pupários de $L$. sativae em folhas de meloeiro coletadas em plantio comercial no munícipio de Floresta-PE no período de 11/04 a 23/05/2017 (área 2).

\begin{tabular}{ccc}
\hline Data da coleta & Eulophidae (\%) & Opius sp. (\%) \\
\hline $11 / 04 / 2017$ & 13,57 & 6 \\
$18 / 04 / 2017$ & 31,48 & 0 \\
$25 / 04 / 2017$ & 35,59 & 15,25 \\
$03 / 05 / 2017$ & 7 & 7,22 \\
$09 / 05 / 2017$ & 21 & 14 \\
$16 / 05 / 2017$ & 24 & 9 \\
$23 / 05 / 2017$ & 50 & 33 \\
\hline Total & 19,95 & 8,17 \\
\hline
\end{tabular}

Época de liberação de parasitoides para controle de L. sativae em meloeiro em Floresta-PE

Como os maiores índices de infestação em ambas as áreas foram verificados no estágio vegetativo da cultura do melão, especificamente entre 14 a 28 D.A.P., recomenda-se, para as condições de Floresta, a liberação dos parasitoides antes desse período, entre a segunda e terceira semana após o plantio.

\section{Conclusões}

Os resultados encontrados mostram que a espécie de mosca-minadora que predomina nos plantios de meloeiro no município de Floresta é a Liriomyza sativae e que a maior infestação dessa praga durante os levantamentos ocorreu entre os dias 14 e 28 após o plantio. Além disso, os parasitoides mais promissores para o controle de L. sativae são os da família Eulophidae, sendo que a sua liberação no campo deve preceder a maior ocorrência da mosca, ou seja, entre a segunda e terceira semana após o plantio.

\section{Referências}


FERRAZ, N. M. G.

Monitoramento de mosca-minadora Liriomyza sp. (Diptera: Agromyzidae) em meloeiro e levantamento de seus inimigos naturais no município de Floresta, Pernambuco

AGROFIT. Agrofit: Sistema de agrotóxicos fitossanitários. Disponível em: http://extranet.agricultura.gov.br/agrofit_cons/principal_agrofit_cons. Acesso em 16 maio. 2015.

ALBA, M. D. A. Leafminers management. In: Biopesticides obtained from plants, another result from coevolution. Current situation and usefulness. Fundación Cajamar, 2014, p. 303 - 328.

ALENCAR, J.A.; HAJI, F.N.P.; ALENCAR, P.C.G.; SILVA NETO, C.R. Dinâmica populacional de Liriomyza sativae em melão em condições semiáridas do Nordeste Brasileiro. In: XIX Congresso Brasileiro de Entomologia., Manaus, Anais do XIX Congresso Brasileiro de Entomologia, Manaus: SEB, 2002. P. 177.

ARAÚJO, E. L.; FERNANDES, D. R. R.; GEREMIAS, L. D.; NETTO, A. C. M.; FILGUEIRA, M. A. Mosca minadora associada à cultura do meloeiro no semiárido do Rio Grande do Norte. Revista Caatinga (Mossoró, Brasil), v.20, n.3, p.210-212. 2007.

AZEVEDO, F. R.; GUIMARÃES, J. A.; TERAO, D.; PINHEIRO NETO, L. G.; FREITAS, J. A. D. Distribuição vertical de minas de Liriomyza sativae Blanchard, 1938 (Diptera: Agromyzidae) em folhas do meloeiro, em plantio comercial. Revista Ciência Agronômica, v.36, n.3, p. 322-326, 2005.

BJORKSTEN, T.A.; ROBINSON, M.; LA SALLE, J. Species composition and population dynamics of leafmining flies and their parasitoids in Victoria. Australian Journal of Entomology (2005) 44, 186191.

CENTRAL SCIENCE LABORATORY, UK, 2004. Protocol for the diagnosis of quarantine organisms: Liriomyza spp. (L. sativae, L. trifolii, L. huidobrensis, L. bryoniae). Disponível em: http://www.fera.defra.gov.uk/plants/plantHealth/pestsdiseases/documents/protocols/liriomyza.pd f. Acesso em: 26 mai. 2015.

COSTA, E. M. Aspectos do manejo integrado da mosca-minadora e conservação de polinizadores em meloeiro. 2016. 79 f. Dissertação (Doutorado em Fitotecnia) - Universidade Federal Rural do Semiárido, Mossoró. 2016.

COSTA LIMA, T. C.; GEREMIAS, L. D.; PARRA, J. R. P. Efeito da temperatura e umidade relativa do ar no desenvolvimento de Liriomyza sativae Blanchard (Diptera: Agromyzidae) em Vigna unguiculata. Neotrop. entomol., Londrina, v. 38, n. 6, p. 727-733, Dec. 2009.

COSTA-LIMA, T. C. Bioecologia e competição de duas espécies de parasitoides neotropicais (Hymenoptera: Braconidae e Eulophidae) de Liriomyza sativae Blanchard, 1938 (Diptera: Agromyzidae). 2011. Tese (Doutorado em Entomologia) - Escola Superior de Agricultura Luiz de Queiroz, Universidade de São Paulo, Piracicaba, 2011. 
FERRAZ, N. M. G.

Monitoramento de mosca-minadora Liriomyza sp. (Diptera: Agromyzidae) em meloeiro e levantamento de seus inimigos naturais no município de Floresta, Pernambuco

COSTA-LIMA, T. C da; SILVA, A. de C; PARRA, J. R. P. Moscas-minadoras do gênero Liriomyza (Diptera: Agromyzidae): aspectos taxonômicos e biologia. Petrolina: Embrapa Semiárido, 2015. (Embrapa Semiárido. Documentos, 268). Disponível em: <https://www.infoteca.cnptia.embrapa.br/infoteca/bitstream/doc/1040146/1/SDC268.pdf>. Acesso em: 13 junho 2020.

COSTA-LIMA, T. C.; COSTA, V. A.; CHAGAS, M. C. M.; PARRA, J. R. P. Perspectives of Liriomyza biological control in Brazil. In: INTERNATIONAL CONGRESS OF ENTOMOLOGY, 25., 2016, Orlando. Abstracts... Annapolis: Entomological Society of America, 2016. Disponível em: https://ainfo.cnptia.embrapa.br/digital/bitstream/item/157294/1/Tiago-2016.pdf. Acesso em: 19 jun. 2020.

CRUZ, C. A.; OLIVEIRA, A. M.; RIBEIRO, L. C.; OLIVEIRA, J. C. L. Ocorrência de parasitismo por Opius sp. (Hymenoptera, Braconidae) no gênero Liriomyza (Diptera: Agromyzidae). Pesquisa em Andamento/PESAGRO, Rio de Janeiro, v. 48, p.1-2, 1988.

FERNANDES, O. A. 2004. Melão - Campo Minado. Revista Cultivar 23: 26-27.

FERREIRA, E. C. B. Estrutura genética de populações naturais de Liriomyza sativae (Diptera: Agromyzidae). 2014. 32 f. Dissertação (Mestrado em Entomologia Agrícola) - Universidade Federal Rural de Pernambuco, Recife.

GUIMARÃES, J. A.; AZEVEDO, F. R.; SOBRINHO, R. B.; MESQUITA, A. L. M. Recomendações para o Manejo das Principais Pragas do Meloeiro na Região do Semi-Árido Nordestino. Fortaleza: Embrapa Agroindústria Tropical, 6 p. (Embrapa Agroindústria Tropical. Comunicado Técnico, 107), 2005.

GUIMARÃES, J. A.; SOBRINHO, R. B.; AZEVEDO, F. R.; ARAUJO, E. L.; TERAO, D.; MESQUITA, A. L. 2008. Manejo Integrado de Pragas do Meloeiro. In: BRAGA SOBRINHO R, GUIMARÃES JA; SOUZA JAD; TERAO D (Org.). Produção Integrada de Melão. Fortaleza, CE: Embrapa Agroindústria Tropical. p. 183-206.

GUIMARÃES, J. A.; FILHO, M. M.; OlIVEIRA, V. R.; LIZ, R. S. de; ARAUJO, E. L. Biologia e manejo de mosca minadora no meloeiro. Comunicação Cientifica EMBRAPA, 2009.

GUIMARÃES, J. A.; OLIVEIRA, V. R.; MICHEREFF, M.; LIZ, R. S. Ocorrência da Mosca minadora sulamericana e seus himenópteros parasitóides em meloeiro no Distrito Federal. Hortic. bras., v. 28, n. 2 (Suplemento - CD Rom), julho 2010. 
FERRAZ, N. M. G.

Monitoramento de mosca-minadora Liriomyza sp. (Diptera: Agromyzidae) em meloeiro e levantamento de seus inimigos naturais no município de Floresta, Pernambuco

HERNÁNDEZ, R.; GUO, K.; HARRIS, M.; LIU, T. X. Effects of selected insecticides on adults of two parasitoid species of Liriomyza trifolii: Ganaspidium nigrimanus (Figitidae) and Neochrysocharis formosa (Eulophidae). Insect Science, v. 18, p. 512-520, 2011.

LA SAlLE, J.; PARRELLA, M. P. 1991. The Chalcidoid Parasites (Hymenoptera: Chalcidoidea) of Economically Important Liriomyza species (Diptera: Agromyzidae) in North America. Proc. Entomol. Soc. Washington, v.93, n. 3, p.571-591, 1991.

LEITE, G. L. D.; PICANÇO, M.; JHAM, G. N.; MARQUINI, F. Intensidade de Tuta absoluta (Meyrick, 1917) (Lepidoptera: Gelechiidae) e Liriomyza spp. (Diptera: Agromyzidae) ataca Lycopersicum esculentum Mill. Folhas. Ciênc. agrotec., Lavras, v. 28, n. 1, p. 42-48, fevereiro de 2004.

LIMA, M. A. A. Resistência de genótipos de meloeiro a mosca-minadora Liriomyza sativae (Blanchard, 1938) (Diptera: Agromyzidae). 2012. 120 f. Tese (Doutorado em Entomologia) Universidade de São Paulo, Piracicaba, 2012.

MURPHY, S.; LA SALLE, J. 1999. Balancing biological control strategies in the IPM of New World invasive Liriomyza leafminers in field vegetable crops. Biocontrol News and Information. Wallingford, v. 20, n. 3, p. 91-104, 1999.

MUSGRAVE, C. A.; POE, S. L.; BENNETT, D. R. Leafminer population estimation in polycultured vegetables. Proc. Fla. State Hortic. Soc, v.88, p.156- 160, 1975.

NASCIMENTO NETO, J. R. Formas de aplicação e doses de nitrogênio e potássio no cultivo de meloeiro amarelo. 2011. 77f. Dissertação (Mestrado em Irrigação e Drenagem) - Universidade Federal do Ceará, Fortaleza, 2011.

PALUMBO, J. C.; MULLIS, C. H.; REYERS, F. J. Composition, seasonal abundance, and parasitism of Liriomyza (Diptera: Agromyzidae) species on lettuce in Arizona. Hortic. Entomol. 87: 1070-1077. 1994.

PARRA, J. R. P P.S.M. BOTELHO, B.S. CORREAA-FERREIRA, J.M.S. BENTO (Eds.) 2002. Controle biológico no Brasil: parasitóides e predadores. São Paulo: Manole, 1-16.

RAMALHO, F. S.; MOREIRA, J. O. T. Algumas moscas-minadoras (Diptera: Agromyzidae) e seus inimigos naturais do trópico semi-árido do Brasil. Ciência e Cultura. V.31, p.8-9, 1979.

RIBEIRO, L. P.; SAUSEN, C. D.; EGEWARTH, R.; STURZA, V. S.; DEQUECH, S. B. Flutuação populacional de Liriomyza sp. (Diptera: Agromyzidae) e parasitoides em cultivo de feijão-de-vagem em estufa 
FERRAZ, N. M. G.

Monitoramento de mosca-minadora Liriomyza sp. (Diptera: Agromyzidae) em meloeiro e levantamento de seus inimigos naturais no município de Floresta, Pernambuco

plástica. In: CONGRESSO DE INICIAÇÃO CIENTíFICA, 16., 2007, Pelotas. Anais... Pelotas: Universidade Federal de Pelotas, 2007. 1 CD-ROM.

SALES JÚNIOR, R.; DANTAS, F. F.; SALVIANO, A. M.; NUNES, G.H.S. Qualidade do melão exportado pelo porto de Natal-RN. Ciência Rural, Santa Maria, v. 36, p.286-289, 2006.

SAS Institute 2001. SAS user's guide: statistics, version 8.2. SAS Institute, Cary, NC computer program, version By SAS Institute.

SILVA, R. K. B. 2008. Técnicas de criação e aspectos do parasitismo de Opius sp. (Hymenoptera: Braconidae) sobre a mosca minadora Liriomyza trifolii (Diptera: Agromyzidae) em meloeiro. Mossoró, Dissertação de Mestrado, UFERSA, 51p.

SPENCER, K. A.; STEYSKAL, G. C. 1986. Manual of the Agromyzidae (Diptera) of the United States. U.S. Department of Agriculture, Agriculture Handbook, n. 638. 478 p.

STRAKHOVA, I. S.; YEFREMOVA, Z. A.; VON TSCHIRNHAUS, M.; YEGORENKOVA, E. N. The parasitoid complex (Hymenoptera, Eulophidae) of leafminer flies (Diptera, Agromyzidae) in the Middle Volga Basin. Entomological Review, v. 93, n. 7, 2013.

WHARTON, R. A. et al. Manual of the new World Genera of the Family Braconidae (Hymenoptera). Special Publications of International Society of Hymenopterists. n. 1, p.439, 1997.

ZHANG, R. J.; YU, D. J.; ZHOU, C. Q. Effect of temperature on certain population parameters of Liriomyza sativae Blanchard (Diptera: Agromyzidae). Entomol Sin 7: 185-192. 2000. 